\title{
Comparative study of the action of fluoroquinolones and vancomycin on biofilms of Staphylococcus strains isolated from infections of peritoneal dialysis catheters
}

\author{
A. Rabhia, b, S. Mahrane ${ }^{a, b}$, S.L. Nouara,b, R. Boushakia,b, A.N. Benkherif \\ Z. Guechia,b, H. Ait Belkacema,c \\ a Central laboratory of biology CHU Hussein Dey Algiers \\ b University Algiers 1 faculty of medicine department of pharmacy \\ c University Algiers 1 faculty of medicine department of medicine
}




\title{
Comparative study of the action of fluoroquinolones and vancomycin on biofilms of Staphylococcus strains isolated from infections of peritoneal dialysis catheters
}

\author{
A. Rabhi, b, S. Mahrane ${ }^{* a, b}$, S.L. Nouara,b, R. Boushakia,b, A.N. Benkherifb, Z. Guechia,b, H. Ait Belkacem ${ }^{a, c}$

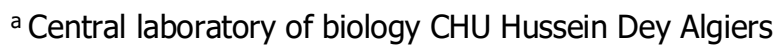 \\ b University Algiers 1 faculty of medicine department of pharmacy \\ ' University Algiers 1 faculty of medicine department of medicine
}

E-mails: yoyoi4400@gmail.com, sadjiamahrane1975@gmail.com, selmanouar@gmail.com, b.radia-microbio@hotmail.com, neilaabir@gmail.com, aitbelle1969@gmail.com

\author{
Received: 5/1/2021 Revised: 30/1/2021 Accepted: 13/3/2021 DOI: https://doi.org/10.31559/CRMI2021.2.1.1
}

\begin{abstract}
:
Background: catheter mediated infections are mainly caused by Staphylococci, the treatment of these infections is challenging because of biofilm formation.

Methods: A microtiter plate biofilm assay was used to study the in vitro activity of Vancomycin, Ofloxacin and Levofloxacin on preformed biofilms of 30 stains of Staphylococcus isolated from peritoneal dialysis catheters and fluids at concentrations like those used intraperitoneally.

Results: The in vitro action of the three antibiotics on preformed biofilms was statistically significant. it was significantly higher for Ofloxacin and Levofloxacin rather than that of Vancomycin.

Discussion: the results demonstrate the effectiveness of the three antibiotics at concentrations like those used intraperitoneally and the superiority of Fluoroquinolones activity on Staphylococcal biofilms, these results correlate with the conclusions of other authors.
\end{abstract}

Keywords: Biofilm; fluoroquinolones; vancomycin; peritoneal dialysis; catheters.

\footnotetext{
${ }^{*}$ Corresponding Author

S.Mahrane

central laboratory of biology CHU Hussein Dey Algiers, university Algiers 1 faculty of medicine department of pharmacy

E-mail: sadjiamahrane1975@gmail.com
} 


\section{Introduction}

Infections occurring in dialysis patients are much more frequent than in general population (100 times) [10]. These infections can compromise the dialysis technique or even be life-threatening; they are the second leading cause of death among dialysis patients [1]. Staphylococci are the most common cause of catheter mediated infections $(45-60 \%$ of cases) [1]. The treatment of these infections is problematic,. in addition to the increased antibiotic resistance of isolated bacterial strains, antibiotic therapy also runs up against the formation of biofilm, especially by staphylococcus strains; various factors have been suggested to be responsible for the resistance of biofilm to antibiotic therapy: slow growth, the presence of an exopolysaccharide matrix that can reduce the diffusion of antibiotics, and the presence of persistent bacteria. [12]

Although vancomycin remains a major antistaphylococcal drug, its action on biofilms remains controversial $[15,11,2],[7,6]$

The aim of this study was to investigate the antibiotic therapy for staphylococcal biofilmsassociated infections by comparing the in vitro action of Fluoroquinolones (Ofloxacin and Levofloxacin) and Vancomycin on a few Staphylococcus isolates obtained from samples of peritoneal dialysis fluids or catheters.

\section{Methods}

We studied 30 isolates of Staphylococcus species (15 Staphylococcus aureus and 15 coagulase negative Staphylococci). These isolates were obtained from samples of peritoneal dialysis fluids or catheters of 24 patients at the HUSSEIN DEY Hospital in Algiers. We evaluated the action of three antibiotics: Vancomycin, Ofloxacin and Levofloxacin on the preformed biofilms.

The choice of antibiotic concentrations was based on the concentrations used by intraperitoneal route according to the recommendations of the International Society for peritoneal dialysis (ISPD) [9], for each antibiotic we opted for the median concentration between the loading dose concentration and the minimum concentration at which the maintenance dose is administered, that minimum concentration is equal to "the concentration of loading dose"-"the maintenance dose concentration" so the concentrations used were calculated by the following formula:

$$
\mathrm{C}=\frac{2 * \text { Cofloadingdose }- \text { Cofmaintenancedose }}{2}
$$

The loading dose concentration for Vancomycin is $1000 \mathrm{mg} / \mathrm{l}$ while the maintenance dose concentration is $25 \mathrm{mg} / \mathrm{l}$, so the concentration of Vancomycin used in this protocol using the formula above is $987.5 \mathrm{mg} / \mathrm{l}$.
The loading dose concentration for Fluoroquinolones is $50 \mathrm{mg} / \mathrm{l}$ while the maintenance dose concentration is $25 \mathrm{mg} / \mathrm{l}$, so the concentration of Fluoroquinolones used in this protocol is 37.5 $\mathrm{mg} / \mathrm{l}$.

\section{Study protocol}

To evaluate the activity of these antibiotics on biofilm, we conducted this evaluation based on microtiter plate biofilm assay protocols proposed by George A. O'Toole [3] and J.H.Merritt et al. (microtiter plate biofilm assay) [4], we opted for the following protocol:

1. Dilution to $1 / 100 \quad(10 \mu \mathrm{l} / 990 \mu \mathrm{l})$ of suspensions (at $0.5 \mathrm{McF}$ ) of each bacterial strain in a liquid BHIB (Brain Heart Infusion Broth) medium.

2. Distribution of $100 \mu \mathrm{l}$ of diluted bacterial suspensions on microplates at a rate of 4 wells per strain for a total of 120 wells for the 30 strains.

3. Incubation of microplates (covered with a film to avoid desiccation) for 24 hours at $37^{\circ} \mathrm{C}$ to induce the formation of biofilms on the polymer of microplates.

4. Three washes were carried out with sterile physiological water to eliminate the planktonic forms of bacteria.

5. For each bacterial strain: a well was incubated with a liquid medium (BHIB) with no additives (control well), a well with a vancomycin solution at $987.5 \mathrm{mg} / \mathrm{l}$ in BHIB, a well with an Ofloxacin solution and a well with a Levofloxacin solution at $37.5 \mathrm{mg} / \mathrm{l}$ in BHIB, at a rate of $100 \mu$ for each solution.

6. Microplates were incubated with these suspensions for 24 Hours at $37 \mathrm{oC}$, avoiding desiccation.

7. Three washes were carried out with sterile physiological water and then the wells were dried at room temperature.

8. The biofilm was quantified using a gentian violet solution at 1\%:150 $\mu \mathrm{l}$ per well with an incubation for 15 minutes.

9. Three washes were carried out to remove the excess of the dye and then the wells were dried for 3 hours at room temperature.

10. $200 \mu \mathrm{l}$ of $95^{\circ}$ ethanol was added for each well.

11. Incubate ethanol in wells for 10 minutes, avoiding desiccation. This will allow the solubilization of gentian violet.

12. The contents of the microplates were transferred to new flat-bottom microplates.

13. Optical Densities (OD) were measured using a plate reader at a wavelength of $595 \mathrm{~nm}$.

14. The measured optical density is correlated with the persistent biofilm mass in each well. 
15. To compare the OD obtained for each one of the 30 strains after the different treatments on the preformed biofilms, we used the statistical Student Test of paired values (on STHDA software), comparing the data in pairs (control/Levofloxacin, vancomycin/Ofloxaccine,...).

\section{Results}

The optical density obtained from each well group according to the applied treatment (Controls, Vancomycin, Ofloxacin, Levofloxacin) for the 30 isolates of Staphylococcus spp are shown in Figure 1 and Table 1.

Table (1): optical densities proportional to biofilm densities of the 30 isolates of Staphylococcus sp before and after different antibiotic treatments.

\begin{tabular}{|c|c|c|c|c|c|c|c|c|}
\hline Strains & 1 & 2 & 3 & 4 & 5 & 6 & 7 & \\
\hline controls & 0,378 & 0,385 & 0,374 & 0,121 & 0,138 & 0,321 & 0,081 & \\
\hline VANCOMYCINE & 0,317 & 0,149 & 0,275 & 0,083 & 0,136 & 0,099 & 0,080 & \\
\hline LEVOFLOXACINE & 0,293 & 0,079 & 0,115 & 0,099 & 0,070 & 0,057 & 0,080 & \\
\hline OFLOXACINE & 0,228 & 0,115 & 0,115 & 0,092 & 0,064 & 0,062 & 0,079 & \\
\hline Strains & 8 & 9 & 10 & 11 & 12 & 13 & 14 & 15 \\
\hline controls & 0,283 & 0,149 & 0,355 & 0,243 & 0,085 & 0,241 & 0,185 & 0,128 \\
\hline VANCOMYCINE & 0,094 & 0,100 & 0,218 & 0,242 & 0,079 & 0,134 & 0,098 & 0,016 \\
\hline LEVOFLOXACINE & 0,137 & 0,148 & 0,154 & 0,241 & 0,076 & 0,085 & 0,094 & 0,110 \\
\hline OFLOXACINE & 0,104 & 0,067 & 0,203 & 0,183 & 0,070 & 0,096 & 0,128 & 0,105 \\
\hline Strains & 16 & 17 & 18 & 19 & 20 & 21 & 22 & 23 \\
\hline controls & 0,101 & 0,257 & 0,141 & 0,151 & 0,168 & 0,247 & 0,188 & 0,141 \\
\hline VANCOMYCINE & 0,100 & 0,209 & 0,140 & 0,144 & 0,131 & 0,210 & 0,180 & 0,140 \\
\hline LEVOFLOXACINE & 0,098 & 0,107 & 0,134 & 0,109 & 0,100 & 0,109 & 0,160 & 0,016 \\
\hline OFLOXACINE & 0,089 & 0,134 & 0,114 & 0,087 & 0,116 & 0,145 & 0,171 & 0,125 \\
\hline Strains & 24 & 25 & 26 & 27 & 28 & 29 & 30 & moyennes \\
\hline controls & 0,145 & 0,133 & 0,085 & 0,116 & 0,130 & 0,130 & 0,101 & 0,190 \\
\hline VANCOMYCINE & 0,082 & 0,113 & 0,084 & 0,097 & 0,089 & 0,101 & 0,100 & 0,135 \\
\hline LEVOFLOXACINE & 0,095 & 0,130 & 0,080 & 0,110 & 0,128 & 0,112 & 0,084 & 0,114 \\
\hline OFLOXACINE & 0,076 & 0,132 & 0,084 & 0,115 & 0,127 & 0,096 & 0,100 & 0,114 \\
\hline
\end{tabular}

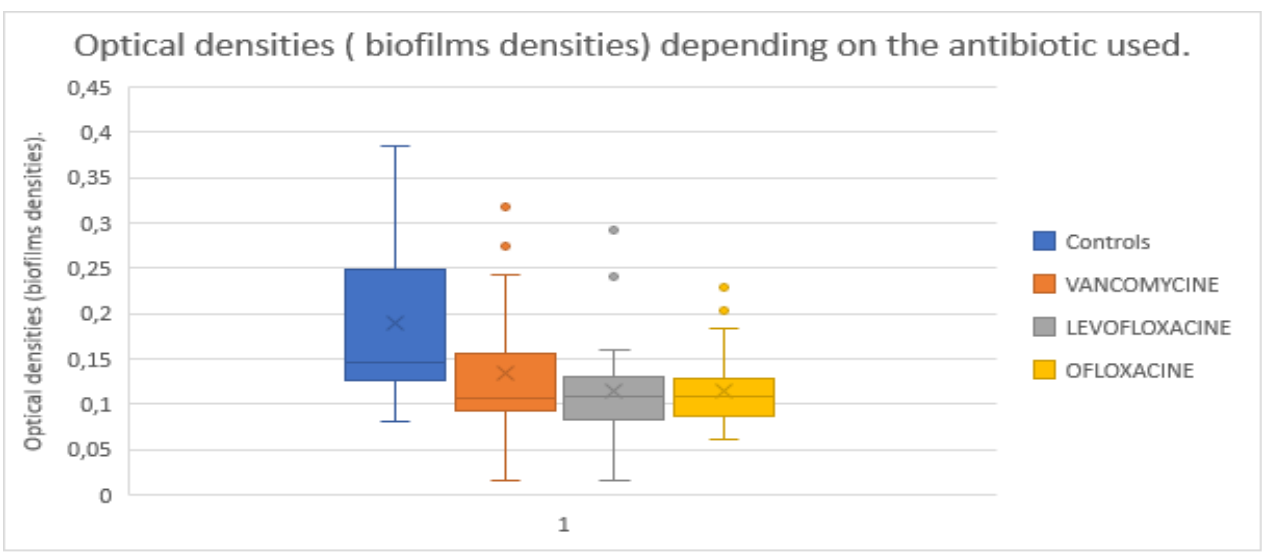

Fig (1): Distribution of OD values in relation to biofilms according to the antibiotic treatment applied. (crosses (X) represent the average OD for each type of well: controls, Vancomycin, Levofloxacin, Ofloxacin. Horizontal lines represent median OD).

The average of optical density for the control well group was 0.190, while for treated wells the average was 0.135 for vancomycin, and 0.114 for Ofloxacin and Levofloxacin.

The action of the 3 antibiotics on the preformed biofilms of the 30 isolates studied was statistically significant (STUDENT test of paired values) compared to controls wells: for vancomycin $\mathrm{p}=000088 \quad(\mathrm{t}=4,5489$, df $=29)$, for ofloxacin $\mathrm{p}=0.000019(\mathrm{t}=5.10, \mathrm{df}=29)$ and for Levofloxacin $p=0.000059(t=4,695, d f=29)$.

The action of Ofloxacin and Levofloxacin on staphylococcal biofilms were significantly higher than that of Vancomycin $\mathrm{p}=0.019(\mathrm{t}=2.47, \mathrm{df}=29)$ and $\mathrm{p}=0.042(\mathrm{t}=2.12, \mathrm{df}=29)$ respectively. On the other hand, the activity of ofloxacin and Levofloxacin on the biofilms of our isolates was similar $p=0.95$ $(\mathrm{t}=0.06, \mathrm{df}=29)$.

\section{Discussion}

A microtiter plate biofilm assay based on the use of a dye and the measurement of optical density to quantify biofilms was used to evaluate the activity of Fluoroquinolones (Ofloxacin and Levofloxacin) and Vancomycin on 30 Staphylococcus sp isolates at concentrations like those used intraperitoneally. The measured optical density at $595 \mathrm{~nm}$ is correlated with the persistent biofilm mass in each well, therefore for each of the isolates: the lower the optical density compared to the control well, the greater the antibiofilm activity of the antibiotic. 
The action of the three antibiotics on the preformed biofilms was statistically significant (STUDENT test of paired values) compared to controls wells. The action of Ofloxacin and Levofloxacin on biofilms were significantly higher than that of Vancomycin, and finally the activity of Ofloxacin and Levofloxacin was similar. The current study confirmed the good action of Fluoroquinolones on preformed biofilms and even the superiority of this activity to that of vancomycin. This result is consistent with the results of Toshihiro and al. [13] which demonstrated better activity of Fluoroquinolones compared to betalactamines and Amikacin on biofilms of Pseudomonas aeruginosa, as well as the study of Tanaka.G and al. [13] which demonstrates the superiority of the effect of Fluoroquinolones on biofilms of Pseudomonas aeruginosa over betalactamines, the studies of Wafi Siala and al.[15] and J.Bauer and al.[5] also affirm the very good activity of Delafloxacin on the biofilms of Staphylococcus aureus. The current study demonstrated a significant anti-biofilm action of Vancomycin in accordance with the results of E.prestreland al.[8], and contrary to the results of R.C.Evans [2].

\section{Conclusion}

The results of our study demonstrate the effectiveness of both Vancomycin and Fluoroquinolones (Ofloxacin and Levofloxacin) and the superiority of the latter on in vitro preformed biofilms of Staphylococcus $s p$ at concentrations like those used intraperitoneally.

\section{References}

1. Beaudreuil S., Hebibi H., Charpentier B. \& Durrbachr A. (2008). Les infections graves chez les patients en dialyse péritonéale et en hémodialyse chronique conventionnelle : péritonites et infections de la voie d'abord vasculaire. Réanimation. 17(3): 233-241, https://doi.org/10.1016/j.reaurg.2008.01.014.

2. Evans R.C. \& Holmes C.J. (1987). Effect of vancomycin hydrochloride on Staphylococcus epidermidis biofilm associated with silicone elastomer. Antimicrobial Agents Chemotherapy, 31(6): 889-894, https://doi.org/10.1128/aac.31.6.889.

3. George A. O'Toole. (2011). Microtiter Dish Biofilm Formation Assay. Journal of Visualized Experiments. (47): 2437, https://doi.org/10.3791/2437.

4. Judith H. Merritt, Daniel E. Kadouri, \& George A. O'Toole. (2005). Growing and AnalyzingStatic Biofilms. Current Protocols in Microbiology. Chap :1, Unit-1B.1.

5. Julia Bauer, Wafi Siala, Paul M. Tulkens \& Françoise Van Bambeke. (2013). A Combined Pharmacodynamic Quantitative and Qualitative
Model Reveals the Potent Activity of Daptomycin and Delafloxacin against Staphylococcus aureus Biofilms. Antimicrobial Agents and Chemotherapy. 57(6): 2726 -2737, https://doi.org/10.1128/aac.00181-13.

6. Owusu-Ababio G. (1999). Effectiveness of ciprofloxacin microspheres in eradicating bacterial biofilm. Journal of Controlled Release. 57(2):151-159, https://doi.org/10.1016/s0168-3659(98)00113-8.

7. Passerini de Rossi B, García C, Calenda M, Vay C \& Franco M. (2009). Activity of levofloxacin and ciprofloxacin on biofilms and planktonic cells of Stenotrophomonas maltophilia isolates from patients with device-associated infections. International Journal of Antimicrobial Agents, 34(3): 260-264, https://doi.org/10.1016/j.ijantimicag.2009.02.022.

8. Prestrel E., Grisold A.J., Reichmann S. \& Hirschl A.M. (2005). ViridansStreptococci in endocarditis and neutropenic sepsis: biofilm formation and effects of antibiotics. Journal of antimicrobial chemotherapy, 55(1): 45-50, https://doi.org/10.1093/jac/dkh479.

9. Recommandations ISPD concernant les infections en dialyse péritonéale. Peritoneal Dialysis International. (2010). 30: 393-423.

10. Sarnak MJ \& Jaber BL. (2000). Mortality caused by sepsis in patients with end-stage renal disease compared with the general population. Kidney International, 58(4):1758-1764 https://doi.org/10.1111/j.1523-1755.2000.00337.x.

11. Sertac Argun Kivanc; Berna Akova Budak; Meral Yildiz \& Merih Kivanc. (2015). The effect of the linezolid and the vancomycine on biofilm production that formed on two different acrylic hydrophobic intraocular lenses. Investigative Ophthalmology \& Visual Science. 56: 287.

12. Stewart PS. (2002) Mechanisms of antibiotic resistance in bacterial biofilms. International Journal of Medical Microbiology, 292(2):107-13, https://doi.org/10.1078/1438-4221-00196.

13. Tanaka G., Shigeta M., Komatsuzawa H., Sugai M., Suginaka H. \&Usui T. (1999). Effect of the Growth Rate of Pseudomonas aeruginosa Biofilms on the Susceptibility to Antimicrobial Agents: $\beta$-Lactams and Fluoroquinolones. Chemotherapy, 45(1):28-36, https://doi.org/10.1159/000007162.

14. Toshihiro Gotoa, Yasuhiko Nakamea, Morio Nishidaa \& Yoshitada Ohia. (1999). In vitro bactericidal activities of beta-lactamases, amikacin, and fluoroquinolones against pseudomonas aeruginosa biofilm in artificial urine. Urology, 53(5): 1058-1062, https://doi.org/10.1016/s0090-4295(98)00649-9.

15. WafiSiala, Marie-Paule Mingeot-Leclercq, Paul M. Tulkens, Marie Hallin, Olivier Denis \& Françoise Van Bambeke. (2014). Comparison of the Antibiotic Activities of Daptomycin, Vancomycin, and the Investigational Fluoroquinolone Delafloxacin against Biofilms from Staphylococcus aureus Clinical Isolates. Antimicrobial Agents and Chemotherapy, 63856397. 